\title{
Radiation dose to the brachial plexus in nasopharyngeal carcinoma treated with intensity-modulated radiation therapy: An increased risk of an excessive dose to the brachial plexus adjacent to gross nodal disease
}

\author{
GUOSHENG FENG ${ }^{1 *}$, HEMING LU², ${ }^{2 *}$ YUAN LIANG ${ }^{3 *}$, HUASHENG CHEN ${ }^{2}$, \\ LIUYANG SHU ${ }^{4}$, SHUI LU ${ }^{4}$, JIANFANG ZHU ${ }^{5}$ and WEIWEI GAO ${ }^{4}$

\begin{abstract}
Departments of ${ }^{1}$ Medical Oncology and ${ }^{2}$ Radiation Oncology, People's Hospital of Guangxi Zhuang Autonomous Region;
${ }^{3}$ Department of Public Health, Guangxi Zhuang Autonomous Region; ${ }^{4}$ Department of Clinical Medicine,

Guangxi Medical University; ${ }^{5}$ Department of Integrated Traditional and Western Medicine,

Guangxi Traditional Chinese Medical University, Nanning, Guanxi 530021, P.R. China
\end{abstract}

Received November 26, 2011; Accepted May 24, 2012

DOI: $10.3892 /$ etm.2012.592

\begin{abstract}
This retrospective study aimed to evaluate the dose to the brachial plexus in patients with nasopharyngeal carcinoma (NPC) treated with intensity-modulated radiation therapy (IMRT). Twenty-eight patients were selected and the brachial plexus was delineated retrospectively. Brachial plexus adjacent/not adjacent to nodes were defined and abbreviated as BPAN and BPNAN, respectively. Dose distribution was recalculated and a dose-volume histogram was generated based on the original treatment plan. The maximum dose to the left brachial plexus was 59.12-78.47 Gy, and the percentage of patients receiving the maximum dose exceeding 60, 66 and 70 Gy was $96.4,57.1$ and $25.0 \%$, respectively; the maximum dose to the right brachial plexus was 59.74-80.31 Gy, and the percentage of patients exposed to a maximum dose exceeding 60, 66 and 70 Gy was 96.4, 64.3 and $39.3 \%$, respectively. For the left brachial plexus, the maximum doses to the BPANs and the BPNANs were 72.84 \pm 3.91 and $64.81 \pm 3.47 \mathrm{~Gy}$, respectively $(\mathrm{p}<0.001)$. For the right brachial plexus, the maximum doses to the BPANs and the BPNANs were 72.91 \pm 4.74 and $64.91 \pm 3.52$ Gy, respectively $(\mathrm{p}<0.001)$. The difference between the left BPANs and the left BPNANs was statistically significant not only for V60 (3.60 vs. $1.01 \mathrm{~cm}^{3}, \mathrm{p}=0.028$ ) but also for V66 (1.26 vs. $\left.0.11 \mathrm{~cm}^{3}, \mathrm{p}=0.046\right)$. There were significant differences in V60 (3.68 vs. $\left.1.16 \mathrm{~cm}^{3}, \mathrm{p}<0.001\right)$ and V66 (1.83 vs. $1.23 \mathrm{~cm}^{3}$, $\mathrm{p}=0.012$ ) between the right BPANs and the right BPNANs. In
\end{abstract}

Correspondence to: Dr Heming Lu, Department of Radiation Oncology, 6 Taoyuan Road, Nanning, Guangxi 530021, P.R. China E-mail: luhming3632@163.com

*Contributed equally

Key words: brachial plexus, nasopharyngeal carcinoma, intensitymodulated radiation therapy, dose distribution conclusion, a large proportion of patients were exposed to the maximum dose to the brachial plexus exceeding the Radiation Therapy Oncology Group-recommended restraints when the brachial plexus was not outlined. The BPANs are at a significantly higher risk of receiving an excessive radiation dose when compared to the BPNANs. A further study is underway to test whether brachial plexus contouring assists in the dose reduction to the brachial plexus for IMRT optimization.

\section{Introduction}

Radiation therapy (RT) as an initial treatment option has long been considered standard practice worldwide for nasopharyngeal carcinoma (NPC) (1). The irradiated volume usually covers the primary site, positive lymph nodes, as well as areas potentially involved by the disease. The whole neck is treated definitively or prophylactically except for patients with N0 stage (2). Critical organs including the parotids, spinal cord, brain stem, pituitary gland, temporal lobes, cranial nerves and middle and inner ears are inevitably exposed to unnecessary irradiation due to their close proximity to the targets. An excessive radiation dose to these organs destroys their partial or whole function, and subsequently impairs the quality of life (QOL) of patients. In addition, toxicities to normal structures often restrict dose escalation to the targets, while insufficient dose to the targets may result in decreased local and/or regional control.

Apart from the organs mentioned above, the brachial plexus is another dose-limiting structure. In laryngeal carcinoma treated with total laryngectomy and left radical neck dissection followed by intensity-modulated radiation therapy (IMRT), the maximum dose to the brachial plexus may reach 68 Gy when not defined as an avoidance structure during treatment planning (3). As a unique subtype of head-andneck cancer, NPC is found to have a higher risk of metastasis to the neck lymph nodes. Nodal involvement in the ipsilateral neck occurs in $85-90 \%$, and in the bilateral neck in $50 \%$ of patients. For this reason, all of these nodal regions are usually 
covered by the clinical target volume, and the tumoricidal radiation dose needs to be delivered to the positive lymph nodes (4). Anatomically, the brachial plexus is in proximity to the gross neck disease at the levels between the C5 and T2 vertebral bodies, thus it is assumed that an increased risk of excessive radiation to the brachial plexus occurs when the neck is treated with a higher dose and no dose constraints is placed on the brachial plexus. Based on this hypothesis, we conducted this retrospective study to evaluate the dose distribution to the brachial plexus in NPC patients treated with definitive IMRT.

\section{Materials and methods}

Study population. From November 2009 to December 2010, 43 patients with newly diagnosed NPC were treated with IMRT at the Department of Radiation Oncology at the People's Hospital of Guangxi Zhuang Autonomous Region. Fifteen patients were excluded from the present study: 5 with distant metastasis, 5 treated with palliative intent due to extensive local or regional disease, 2 treated with palliative intent due to uncontrolled medical comorbidities, 3 treated with conventional RT followed by IMRT. The remaining 28 treated with definitive IMRT were included for analysis. Of these patients, 20 were men and 8 were women with a median age of 42 years (range, 22-76). All patients had histologically confirmed undifferentiated carcinoma. The staging distribution according to the 2002 AJCC Staging System is listed in Table I. The study was approved by the Institutional Review Board (IRB) of the People's Hospital of Guangxi Zhuang Autonomous Region.

Intensity-modulated radiotherapy. Patients were immobilized in a supine position with the head in a neutral position, with a tailored thermoplastic mask covering the head, neck and shoulders. Intravenous contrast-enhanced CT using 2-mm slices from the vertex to the manubriosternal joint was performed for planning. The CT data were imported to the CMS-XiO treatment planning system (CMS Inc., St. Louis, MO, USA).

The target delineation for NPC patients has been described previously (5). In brief, the primary gross tumor volume (GTVnx) and the involved lymph nodes (GTVnd) included all known gross disease as determined by the imaging, clinical, and endoscopic findings. Clinical target volume (CTVnx) included the GTVnx plus a 5- to 10-mm margin, and CTVnd included the GTVnd plus a 5-mm margin. CTV1 was defined as the entire nasopharynx, parapharyngeal space, pterygopalatine fossa, posterior third of the nasal cavity and maxillary sinuses, inferior sphenoid sinus, posterior ethmoid sinus, skull base, and anterior half of the clivus. CTV1 also included bilateral retropharyngeal lymph nodes and ipsilateral level II for node-negative neck. CTV1 extended to the next ipsilateral level for node-positive neck, or included the full length of ipsilateral neck for node-positive in the lower neck. CTV2 was defined as low-risk node region below the CTV1. Level V was separated by the borderline between the CTV1 and CTV2 (i.e. regions above the borderline were covered by the CTV1 and regions below the borderline were covered by the CTV2). Level Ib was not included unless the ipsilateral level II was involved. The respective planning target volumes (PTVs) were generated with a 3-mm margin when daily kilovoltage cone-
Table I. Baseline characteristics and treatment details.

\begin{tabular}{|c|c|}
\hline Characteristic & P-value \\
\hline \multicolumn{2}{|l|}{ Age (years) } \\
\hline Median & 42 \\
\hline Range & $22-76$ \\
\hline \multicolumn{2}{|l|}{ Gender, no. (\%) } \\
\hline Male & $20(71.4)$ \\
\hline Female & $8(28.6)$ \\
\hline \multicolumn{2}{|l|}{ T stage, no. (\%) } \\
\hline $\mathrm{T} 1$ & $2(7.1)$ \\
\hline $\mathrm{T} 2$ & $13(46.4)$ \\
\hline T3 & $8(28.6)$ \\
\hline $\mathrm{T} 4$ & $5(17.9)$ \\
\hline \multicolumn{2}{|l|}{ N stage, no. (\%) } \\
\hline N0 & $6(21.5)$ \\
\hline N1 & $10(35.7)$ \\
\hline N2 & $9(32.1)$ \\
\hline N3 & $3(10.7)$ \\
\hline \multicolumn{2}{|l|}{ AJCC stage group, no. (\%) } \\
\hline IIA & $1 \quad(3.6)$ \\
\hline IIB & $4(14.3)$ \\
\hline III & $15(53.5)$ \\
\hline IVA & $5(17.9)$ \\
\hline IVB & $3(10.7)$ \\
\hline \multicolumn{2}{|c|}{ Radiation dose (Gy), median (range) } \\
\hline Median dose to PTVnx & $70.0(66.0-71.6)$ \\
\hline Median dose to PTVnd & $69.0(63.6-70.0)$ \\
\hline Median dose to PTV1 & $60.0(56.1-64.0)$ \\
\hline Median dose to PTV2 & $54.0(50.4-56.1)$ \\
\hline
\end{tabular}

beam computed tomography (KV-CBCT) was performed, or with a 4-mm margin when not. The contoured critical structures included the brain stem, chiasm, optic nerves, spinal cord, eyes, lens, cochlea, parotid glands, oral cavity, larynx, mandible and temporomandibular joints. The brachial plexus was not delineated during the initial planning, and dose to the brachial plexus was not calculated.

The treatment plans were optimized by using the CMS inverse treatment-planning system. The median doses delivered to the PTVnx, PTVnd, PTV1, and PTV2 were 70.0 Gy (66.0-71.6 Gy), 69.0 (63.6-70.0 Gy), 60.0 (56.1-64.0 Gy) and 54.0 Gy (50.4-56.1 Gy), respectively, in 30-33 fractions. All patients were treated once daily, with 5 fractions weekly (Table I). The dose constrains to critical structures were within the tolerance, according to the Radiation Therapy Oncology Group (RTOG) 0225 protocol, and every effort was made to meet the criteria as closely as possible. IMRT was delivered via seven fixed-gantry angles with an Elekta Synergy Linear Accelerator (Elekta Ltd).

Concurrent chemotherapy. Two patients were treated with IMRT alone and 26 were treated with IMRT and concurrent platinum-based chemotherapy. Of these, 18 patients received platinum alone and 8 patients received combination chemotherapy with platinum and 5-fluorouracil. 
Table II. Mean volume of, and dose to the brachial plexus.

\begin{tabular}{|c|c|c|c|c|}
\hline & $\begin{array}{c}\text { Left side } \\
(\text { mean } \pm \mathrm{SD})\end{array}$ & $\begin{array}{c}\text { Right side } \\
(\text { mean } \pm \mathrm{SD})\end{array}$ & $\begin{array}{l}\text { Difference in the mean } \\
\qquad(95 \% \mathrm{CI})\end{array}$ & P-value \\
\hline Mean volume of $\mathrm{BP}\left(\mathrm{cm}^{3}\right)$ & $6.02 \pm 2.78$ & $6.17 \pm 1.90$ & $-0.15(-1.63-1.32)$ & 0.837 \\
\hline \multicolumn{5}{|l|}{ Dose to BP (Gy) } \\
\hline Max & $66.82 \pm 4.99$ & $67.77 \pm 5.53$ & $-0.95(-3.77-1.87)$ & 0.502 \\
\hline Min & $22.43 \pm 4.87$ & $22.36 \pm 15.35$ & $0.07(-8.03-8.16)$ & 0.067 \\
\hline Mean & $50.56 \pm 15.10$ & $51.70 \pm 15.07$ & $-1.14(-9.23-6.94)$ & 0.778 \\
\hline
\end{tabular}

$\mathrm{BP}$, brachial plexus.

Brachial plexus contour and dose recalculation. The left and right brachial plexi were delineated retrospectively and separately on the initial axial-planning CT scan. A delineation was performed step-by-step, following the CT-based atlas for delineating the brachial plexus, a guideline proposed by Hall et al (6) and endorsed by the RTOG.

To avoid inter-observer variations, a senior radiation oncologist was assigned to contour the brachial plexus. After completing the delineation of the brachial plexus, the dosedistribution was recalculated and a dose-volume histogram was generated based on the original treatment plan, without changes in any dosimetric parameters. The mean volume of, and the dose to the brachial plexus were compared between the left and right side. Upon delineation of the brachial plexus, it was determined whether or not the brachial plexus was adjacent to the positive lymph nodes. BPAN was defined when the ipsilateral neck from C5 to T2 vertebral body consisted of positive lymph nodes, whereas BPNAN was defined when no positive lymph nodes were present. The BPANs were compared with the BPNANs with respect to their mean dose, maximum dose and irradiated volume exceeding different dose levels.

Statistical analysis. Independent-samples t-test was used to test the mean differences in each parameter (i.e. mean volume of the brachial plexus between the right and left sides, dose to the brachial plexus, mean and maximum doses to the BPANs and BPNANs and irradiated volumes of the brachial plexus at different dose levels). All statistical tests were 2-sided, and $\mathrm{p} \leq 0.05$ was considered statistically significant. Analyses were performed using Microsoft Office Excel (Version 2007) and SPSS software (SPSS 17.0, SPSS, Inc., Chicago, IL, USA).

\section{Results}

The mean volume of, and the dose to the brachial plexus. The mean volumes were $6.02 \pm 2.78 \mathrm{~cm}^{3}$ for the left brachial plexus and $6.17 \pm 1.90 \mathrm{~cm}^{3}$ for the right $(\mathrm{p}=0.837)$. The maximum dose to the left brachial plexus ranged from 59.12 to $78.47 \mathrm{~Gy}$. The percentage of patients receiving the maximum dose to the left brachial plexus exceeding 60, 66 and 70 Gy was 96.4 (27/28), 57.1 (16/28) and $25.0 \%$ (7/28), respectively, whereas the maximum dose to the right brachial plexus ranged from 59.74 to $80.31 \mathrm{~Gy}$. The percentage of patients receiving the maximum dose to the right brachial plexus exceeding 60,66 and 70 Gy was $96.4(27 / 28), 64.3(18 / 28)$ and $39.3(11 / 28)$,

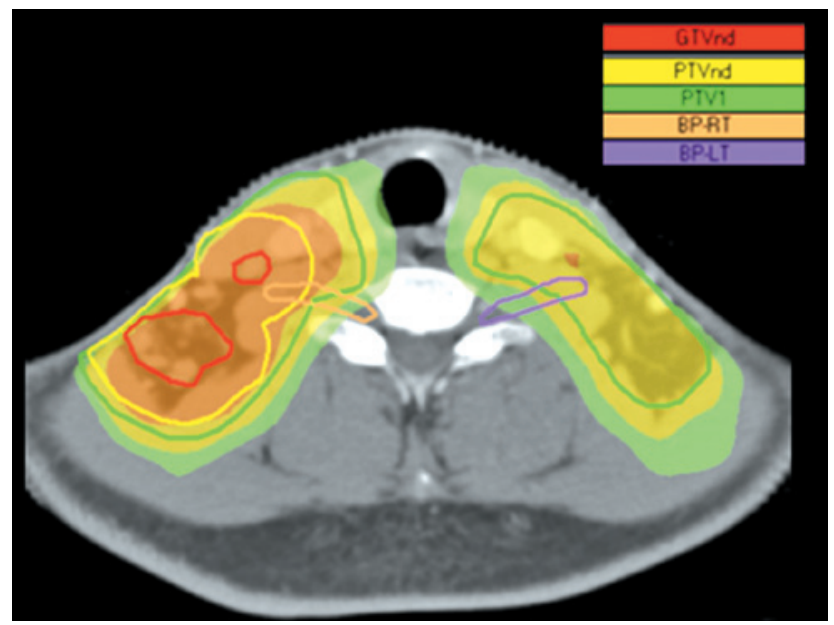

Figure 1. Radiation dose distribution to the brachial plexus in a patient with T2bN3M0 disease. Dose prescription for PTVnd and PTV1 is 68.20 (orange color wash) and 59.20 Gy (yellow color wash), respectively, delivered in 31 fractions. Note that the right brachial plexus is adjacent to the metastatic lymph nodes and receives a maximum dose as high as the prescription dose to the PTVnd (68.20 Gy).

respectively. The minimum and mean doses to the brachial plexus for the left side exhibited no significant differences compared with the corresponding doses to the brachial plexus for the right side (Table II). A typical example of the dosedistribution to the brachial plexus in a patient with T2bN3M0 disease is shown in Fig. 1.

Mean and maximum doses to the BPANs and BPNANs. We identified 16 BPANs, which consisted of 7 BPANs on the left side and 9 BPANs on the right side. We also identified 40 BPNANs, including 21 BPNANs on the left side and 19 BPNANs on the right side. For the left brachial plexus, the maximum doses to the BPANs and the BPNANs were $72.84 \pm 3.91$ and $64.81 \pm 3.47 \mathrm{~Gy}$, respectively $(\mathrm{p}<0.001)$. For the right brachial plexus, the maximum doses to the BPANs and the BPNANs were $72.91 \pm 4.74$ and $64.91 \pm 3.52 \mathrm{~Gy}$, respectively $(\mathrm{p}<0.001)$. No significant differences were found in the mean doses to the BPANs and the BPNANs for either side (Table III).

Irradiated volume of the brachial plexus at different dose levels. V40, V50, V60 and V66 represent the percentage of a specific structure exceeding 40,50,60 and $66 \mathrm{~Gy}$, respectively. 
Table III. Mean and maximum doses to the BPANs and BPNANs.

\begin{tabular}{|c|c|c|c|c|}
\hline & $\begin{array}{c}\text { BPAN } \\
(\text { mean } \pm \text { SD) }\end{array}$ & $\begin{array}{c}\text { BPNAN } \\
(\text { mean } \pm \text { SD) }\end{array}$ & $\begin{array}{c}\text { Difference in mean } \\
\quad(95 \% \mathrm{CI})\end{array}$ & P-value \\
\hline \multicolumn{5}{|c|}{ Dose to left-sided BP (Gy) } \\
\hline Mean & $54.62 \pm 17.48$ & $49.20 \pm 14.44$ & $5.42(-8.21-19.05)$ & 0.421 \\
\hline $\operatorname{Max}$ & $72.84 \pm 3.91$ & $64.81 \pm 3.47$ & $8.03(4.82-11.24)$ & $<0.001$ \\
\hline \multicolumn{5}{|c|}{ Dose to right-sided BP (Gy) } \\
\hline Mean & $57.34 \pm 11.75$ & $48.57 \pm 16.07$ & $8.77(-3.16-20.71)$ & 0.143 \\
\hline Max & $72.91 \pm 4.74$ & $64.91 \pm 3.52$ & $8.01(4.77-11.24)$ & $<0.001$ \\
\hline
\end{tabular}

BP, brachial plexus; BPAN, brachial plexus adjacent to node; BPNAN, brachial plexus nonadjacent to node.

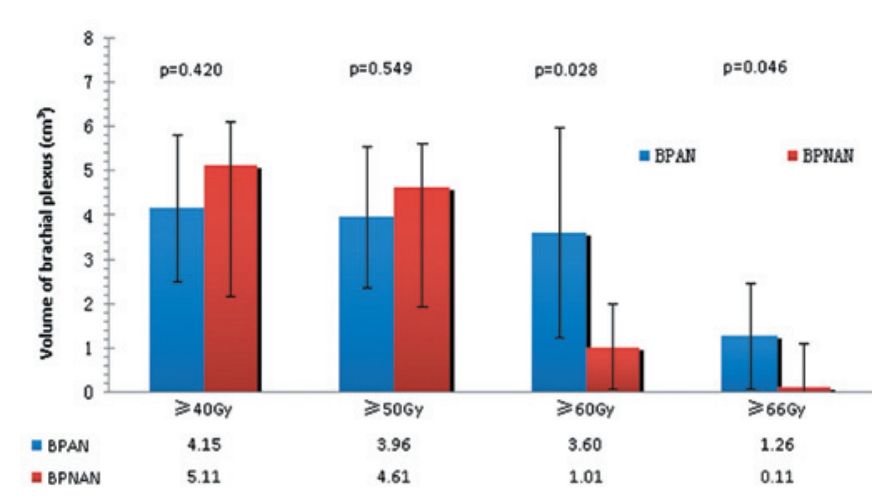

Figure 2. Irradiated volume of the left brachial plexus at different dose levels.

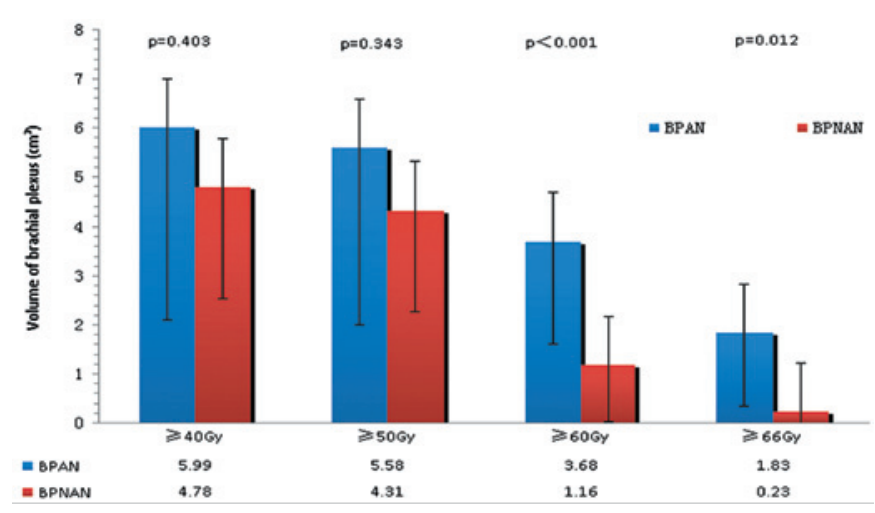

Figure 3. Irradiated volume of the right brachial plexus at different dose levels.
In the present study, absolute volumes were used for analyzing the irradiated volume of the brachial plexus at different dose levels. As shown in Figs. 2 and 3, there were no significant differences in V40 and V50 between the BPANs and BPNANs. V40 for the left BPANs was $4.15 \mathrm{~cm}^{3}$, whereas for the left BPNANs, it was $5.11 \mathrm{~cm}^{3}(\mathrm{p}=0.420)$. V50 for the left BPANs was $3.96 \mathrm{~cm}^{3}$, whereas for the left BPNANs it was $4.61 \mathrm{~cm}^{3}$ ( $\mathrm{p}=0.549)$. V40 was $5.99 \mathrm{~cm}^{3}$ for the right BPANs and $4.78 \mathrm{~cm}^{3}$ for the right BPNANs ( $\mathrm{p}=0.403)$. V50 was $5.58 \mathrm{~cm}^{3}$ for the right BPANs and $4.31 \mathrm{~cm}^{3}$ for the right BPNANs $(\mathrm{p}=0.343)$. By contrast, the difference between the left BPANs and BPNANs was statistically significant not only for V60 (3.60 vs. $\left.1.01 \mathrm{~cm}^{3}, \mathrm{p}=0.028\right)$ but also for V66 (1.26 vs. $0.11 \mathrm{~cm}^{3}$, $\mathrm{p}=0.046)$. Similar results were found on the right side. There were significant differences in V60 (3.68 vs. $1.16 \mathrm{~cm}^{3}, \mathrm{p}<0.001$ ) and V66 (1.83 vs. $\left.1.23 \mathrm{~cm}^{3}, \mathrm{p}=0.012\right)$ between the right BPANs and BPNANs.

\section{Discussion}

Injuries to the brachial plexus associated with RT have been documented in various cancer types, including breast cancer (7), lung cancer (8) and head-and-neck cancer (9). The brachial plexopathy is often gradually deteriorated and no effective treatment is available. Thus, the major aim of RT is to diminish or, at best, to avoid an excessive radiation dose to the brachial plexus. With the rapid evolvement of radiation therapy techniques during the last decade, IMRT has been widely accepted as the preferential technique for treating head-andneck cancer. It is generally believed that IMRT is superior to conventional RT with respect to local and/or regional tumor control and critical organ sparing. However, in a recent study, Chen et al (10) found that the dose to the brachial plexus was significantly increased among patients with head-and-neck cancer undergoing IMRT compared with conventional RT when no brachial plexus constraint was used. Contouring the brachial plexus as an avoidance structure during treatment planning may minimize the radiation dose to this region.

The brachial plexus starts in the posterior triangle of the neck and travels distally into the upper extremity, where it divides into rami, trunks, divisions, cords, and terminal nerve branches. The anatomic location of the brachial plexus is the area of the thoracic outlet, between the first rib and the clavicle. The brachial plexus extends from the lateral border of the scalene anterior muscle to the caudal border of the pectoralis minor (11). An accurate delineation of the brachial plexus is a prerequisite for achieving brachial plexus-sparing during the planning phase. Hall et al (6) proposed a standard method for contouring the brachial plexus on a treatment planning CT scan. Although the method used anatomic landmarks on axial CT images as a surrogate for identifying the location of the brachial plexus, it did provide a reliable set of guidelines for the consistent contouring of the brachial plexus (12). Magnetic resonance (MR) imaging can help identify the anatomy of the brachial 
plexus. Fusion CT-MR imaging facilitates the delineation, even though its routine application is restricted due to unavailability at most radiation oncology facilities and other reasons, such as difference in patient positioning when performing $\mathrm{CT}$ imaging and MR imaging (13). Therefore, CT imaging alone remains the widely accepted tool for contouring the brachial plexus to date. By following the instructions, we delineated the brachial plexus in 28 NPC patients. Unlike others, we separated the left- and the right-sided brachial plexus when delineating. The mean volumes between the left and right brachial plexus had no significant difference, indicating that this structure has bilateral symmetry. In addition, no significant differences were noted in the maximum, minimum and the mean dose to the brachial plexus between the two sides.

Due to the high incidence of cervical nodal metastasis, the entire neck - including the retropharyngeal nodes and levels I-V lymph nodes - is considered to be at risk for involvement. Radical radiation delivered to the gross nodal disease may increase the risk of an excessive dose to the brachial plexus. In the current study, we found that the BPANs received a significantly higher maximum dose than the BPNANs (72.84 \pm 3.91 vs. $64.81 \pm 3.47$ Gy on the left side, $p<0.001$; $72.91 \pm 4.74$ vs. $64.91 \pm 3.52$ Gy on the right side, $p<0.001$ ), reflecting the close proximity of nodal regions to the brachial plexus. In fact, although the brachial plexus did not abut the lymph nodes, its maximum dose was close to the target dose (Table III). In addition, for the brachial plexus on both sides, the BPANs had significant differences in V60 and V66, compared with the BPNANs. The findings are similar to the results of other researchers. Millender et al (14) retrospectively evaluated the radiation dose to the brachial plexus in 16 patients with head-and-neck cancer treated with extended field IMRT technique and found that the median maximum-point dose was higher when the plexus was adjacent to grossly positive nodes (71.9 Gy) than when it was adjacent to node-negative regions ( $65.7 \mathrm{~Gy})$. The median volumes receiving a dose greater than $60 \mathrm{~Gy}$ were 2.95 and $0.84 \mathrm{cc}$, respectively.

Dose constraints to the brachial plexus suggested by the Radiation Therapy Oncology Group (RTOG) in several protocols (RTOG 0435, RTOG 0522, and RTOG 0615) range from 60 to 66 Gy at 2 Gy per fraction. McGary et al (3) found, that doses to the brachial plexus approximated the target dose when the brachial plexus was not defined as an avoidance structure and the target dose was set to 66 or $70 \mathrm{~Gy}$. A hot spot could be seen in the brachial plexus that exceeded 70 Gy. In our study, $96.4 \%$ of the brachial plexus on both sides had a maximum dose exceeding $60 \mathrm{~Gy}$, and the percentages of patients receiving the maximum dose exceeding 66 Gy were 57.1 and $64.3 \%$ for the left and right plexus, respectively. These findings indicate that for the majority of NPC patients the maximum dose to the brachial plexus will be beyond the recommended constraints, when no brachial plexus is delineated as a restricted volume.

There were no data available on the incidence of brachial plexopathy for our patients since the study was conducted retrospectively and patients had no regular follow-up. Nevertheless, to our knowledge, the present report represents the first attempt to analyze the radiation dose to the brachial plexus among patients treated solely for nasopharyngeal carcinoma.

In conclusion, a large proportion of patients are exposed to the maximum dose to the brachial plexus exceeding the
RTOG recommended restraints, when the brachial plexus is not outlined. For the brachial plexus on both sides, the BPANs had higher volumes in V60 and V66 than the BPNANs. The BPANs received a significantly higher maximum dose than that of the BPNANs. A further study is underway to assess whether brachial plexus contouring assists in dose reduction to the brachial plexus for IMRT optimization.

\section{Acknowledgements}

The present study was financed by grants from the Sci-Tech Office of the Guangxi Zhuang Autonomous Region, China (nos. 0816004-40 and 099300B-6).

\section{References}

1. Lu H, Peng L, Yuan X, Hao Y, Lu Z, Chen J, Cheng J, Deng S, Gu J, Pang Q and Qin J: Concurrent chemoradiotherapy in locally advanced nasopharyngeal carcinoma: a treatment paradigm also applicable to patients in Southeast Asia. Cancer Treat Rev 35: 345-353, 2009.

2. Gao Y, Zhu G, Lu J, Ying H, Kong L, Wu Y and Hu C: Is elective irradiation to the lower neck necessary for N0 nasopharngeal carcinoma ? Int J Radiat Oncol Biol Phys 77: 1397-1402, 2010.

3. McGary JE, Grant WH, Teh BS, Paulino AC and Butler E: Dosimetric evaluation of the brachial plexus in the treatment of head and neck cancer. Int J Radiat Oncol Biol Phys 69 (Suppl 3): S464-S465, 2007.

4. Lo SS and Lu JJ: Natural history, presenting symptom, and diagnosis of nasopharyngeal carcinoma. In: Nasopharyngeal Cancer-Multidisciplinary Management. Lu JJ, Cooper JS and Lee AWM (eds). Springer-Verlag, Berlin Heidelberg, p46, 2009.

5. Lu H, Chen J, Huang B, Cheng J, Peng L, Hao Y, Liao C, Mo Y, Wu D and Qin J: Feasibility and efficacy study of weekly cisplatin with concurrent intensity-modulated radiation therapy for nasopharyngeal carcinoma - preliminary results. Oral Oncol 46: 743-747, 2010.

6. Hall W, Guiou M, Lee N, Dublin A, Narayan S, Vijayakumar S, Purdy JA and Chen AM.: Development and validation of a standarlized method for contouring the brachial plexus: preliminary dosimetric analysis among patients treated with IMRT for headand-neck cancer. Int J Radiat Oncol Biol Phys 72: 1362-1367, 2008.

7. Johansson S, Svensson H and Denekamp J: Timescale of evolution of late radiation injury after postoperative radiotherapy of breast cancer patients. Int J Radiat Oncol Biol Phys 48: 745-750, 2000.

8. Chamberlain DD, Rowe BP and Decker RH: Simultaneous treatment of synchronous primary lung cancers with stereotactic body radiotherapy (SBRT). Int J Radiat Oncol Biol Phys 75 (Suppl 1): S470, 2009.

9. Chen AM, Hall W, Guiou M, Mathai M, Vijayakumar S and Purdy JA: Brachial plexopathy after radiation therapy for headand-neck cancer. Int J Radiat Oncol Biol Phys 75 (Suppl 1): S31-S32, 2009.

10. Chen AM, Hall WH, Li BQ, Guiou M, Wright C, Mathai M, Dublin A and Purdy JA: Intensity-modulated radiotherapy increases dose to the brachial plexus compared with conventional radiotherapy for head-and-neck cancer. Br J Radiol 84: 58-63, 2011.

11. Leinberry CF and Wehbé MA: Brachial plexus anatomy. Hand Clin 20: 1-5, 2004.

12. Yi SK, Hall WM, Mathai M, Dublin AB, Gupta V, Purdy JA and Chen AM: Validating the RTOG-endorsed brachial plexus contouring atlas: an evaluation of reproducibility among patients treated by intensity-modulated radiotherapy for head-and-neck cancer. Int J Radiat Oncol Biol Phys 82: 1060-1064, 2011.

13. Truong MT, Nadgir RN, Hirsch AE, Subramaniam RM, Wang JW, Wu R, Khandekar M, Nawaz AO and Sakai O: Brachial plexus contouring with CT and MR imaging in radiation therapy planning for head and neck cancer. Radiographics 30: 1095-1103, 2010.

14. Millender LE, Bucci MK, Quivey JM, Chin CT and Xia P: Evaluation of dose to the brachial plexus using intensitymodulated radiation therapy for treatment of head and neck cancer. Int J Radiat Oncol Biol Phys 60 (Suppl 1): S505, 2004. 